\title{
Hardware Performance Counter-Based Malware Identification and Detection with Adaptive Compressive Sensing
}

\author{
XUEYANG WANG, New York University \\ SEK CHAI, MICHAEL ISNARDI, and SEHOON LIM, SRI International \\ RAMESH KARRI, New York University
}

\begin{abstract}
Hardware Performance Counter-based (HPC) runtime checking is an effective way to identify malicious behaviors of malware and detect malicious modifications to a legitimate program's control flow. To reduce the overhead in the monitored system which has limited storage and computing resources, we present a "sample-locally-analyze-remotely" technique. The sampled HPC data are sent to a remote server for further analysis. To minimize the I/O bandwidth required for transmission, the fine-grained HPC profiles are compressed into much smaller vectors with Compressive Sensing. The experimental results demonstrate an 80\% I/O bandwidth reduction after applying Compressive Sensing, without compromising the detection and identification capabilities.
\end{abstract}

\section{CCS Concepts: • Security and privacy $\rightarrow$ Intrusion/anomaly detection and malware mitigation}

Additional Key Words and Phrases: Hardware performance counters, compressive sensing, malware identification and detection

ACM Reference Format:

Xueyang Wang, Sek Chai, Michael Isnardi, Sehoon Lim, and Ramesh Karri. 2016. Hardware performance counter-based malware identification and detection with adaptive compressive sensing. ACM Trans. Archit. Code Optim. 13, 1, Article 3 (March 2016), 23 pages.

DOI: http://dx.doi.org/10.1145/2857055

\section{INTRODUCTION}

The increasing complexity of modern computer systems results in the increase of security vulnerabilities, making computer systems appealing targets for attacks. A program running on a processor can be compromised in several ways. For example, the program binaries can be corrupted or the program binaries originating from an untrusted source are executed. A more dangerous type of attacks changes the control flow of the program at runtime, leading to arbitrary code execution in the victim system. There has been a long line of research on defending against runtime attacks [Lie et al. 2000; Suh et al. 2005; Fiskiran and Lee 2004; Abadi et al. 2005].

Hardware events-based checking is a new type of runtime malware detection technique which can detect malicious modifications to a program's control-flow with a low cost. Several hardware events-based techniques have been proposed to detect malware,

Authors' addresses: X. Wang and R. Karri, Department of Electrical and Computer Engineering, Tandon School of Engineering, New York University, Brooklyn, NY, 11201; email: \{xueyang.wang, rkarri\}@nyu.edu; S. Chai, M. Isnardi, and S. Lim, SRI International, Princeton, NJ, 08540; email: \{sek.chai, michael.isnardi, sehoon.lim\}@sri.com.

Permission to make digital or hard copies of part or all of this work for personal or classroom use is granted without fee provided that copies are not made or distributed for profit or commercial advantage and that copies show this notice on the first page or initial screen of a display along with the full citation. Copyrights for components of this work owned by others than ACM must be honored. Abstracting with credit is permitted. To copy otherwise, to republish, to post on servers, to redistribute to lists, or to use any component of this work in other works requires prior specific permission and/or a fee. Permissions may be requested from Publications Dept., ACM, Inc., 2 Penn Plaza, Suite 701, New York, NY 10121-0701 USA, fax +1 (212) 869-0481, or permissions@acm.org.

(c) 2016 ACM 1544-3566/2016/03-ART3 $\$ 15.00$

DOI: http://dx.doi.org/10.1145/2857055 
both in user space and kernel space [Malone et al. 2011; Demme et al. 2013; Wang and Karri 2013]. The key idea of the hardware events-based checking is that the behavior of a program can be characterized with the total occurrences of specified hardware events during its execution, and the relationship between the occurrences of different monitored events. Such hardware events include retired instructions, branches, returns, floating point operations, and so on. These monitored hardware events are automatically and securely counted by a set of special-purpose registers called Hardware Performance Counters (HPC), which exist in most modern processors. By using HPCs, the checking cost is significantly reduced, and the tamper-resistance is enhanced. HPCs can be leveraged for different types of malware detection and identification: static or dynamic and anomaly based or signature based.

There are several advantages to HPC-based detection techniques, making them more secure and efficient than antivirus software and other existing runtime integrity checking techniques. First, because an antivirus system is itself software, it is vulnerable to software-based attacks [Xue 2008]. Bugs or oversights in the antivirus software or underlying system software can be exploited to disable the protection. HPCs are inaccessible to malicious software, which increases the tamper resistance. Second, most antivirus software and runtime integrity checking techniques typically use static characteristics of malware such as suspicious strings of instructions in the binary to detect threats. It is relatively easy for malware writers to bypass static analysis by producing many different code variants that are functionally equivalent. HPC-based detection characterizes a program with its actual behavior. To perform a malicious action, no matter how the malware writers change the code, the behavior of the malware cannot be modified too much.

Though having very low overhead on data acquirement, the current techniques bring nonnegligible overhead on data storage and analysis. An HPC-based check normally requires a comparison between the runtime HPC profile and a pregenerated reference. For anomaly-based techniques, the reference is an HPC profile of a clean module; for signature-based techniques, the reference is an HPC profile of a malicious behavior. A local database is required on every monitored platform to store the HPC profile of each clean software module and that of each known malicious behavior. The size of the required storage will increase when dynamic checking is applied (HPCs are periodically sampled and logged). Moreover, when new types of malware are discovered, the database on each platform needs to be updated which further increases the storage size and introduces extra maintenance cost.

Besides the storage overhead, performing the data classification and matching for malware detection and identification is a compute-intensive task and thus will also cause significant performance overhead. Because of these challenges, the current HPCbased techniques are inapplicable to embedded systems with very limited storage and computing resources, such as smart power meters in the customer premise [EEI-AEICUTC 2011] and remote terminal units in the power grid [Syal and Ofei-Amoh 2013], even if they already use HPC-enable microprocessors.

To reduce the storage and performance overhead in the monitored system, we propose an architecture which uses a "sample-locally-analyze-remotely" dynamic checking. The detection and identification are performed in a remote server, instead of running both data collection and analysis locally on the monitored platform. To lower the I/O bandwidth devoted to sending collected HPC data from the monitored platform to the remote analyzer, we employ the Compressive Sensing [Baraniuk 2007] technique to generate a compressed version of HPC data before sending it out. The compressed HPC data is accurately recovered in the remote server for further analysis. 
We make the following contributions in this article:

-Propose a remote malware detection and identification architecture which reduces the storage and performance overhead in the monitored systems, making the HPC-based malware detection and identification applicable to microprocessor-based embedded platforms with very limited storage and computing resources.

- Leverage the Compressive Sensing technique to significantly reduce the I/O bandwidth of the proposed architecture. The results show an $80 \% \mathrm{I} / \mathrm{O}$ bandwidth reduction after applying Compressive Sensing, without compromising the detection and identification capabilities.

The rest of this article is organized as follows: Section 2 describes the exiting HPCbased malware detection techniques and discusses their limitations. Section 3 gives the overview of the proposed technique. Section 4 presents the implementation details. The evaluation results are shown in Section 5. Section 6 lists the related work. The conclusion and future work are presented in Section 7.

\section{HPC-BASED MALWARE DETECTION}

\subsection{Introduction to HPCs}

HPCs are a set of special-purpose registers built into a modern microprocessor's performance monitoring unit (PMU) to store the counts of hardware-related activities. HPCs were originally designed for performance debugging of complex software systems. They work along with event selectors which specify the monitored hardware events and the digital logic which increases a counter after a hardware event occurs. Relying on HPCbased profilers, the developers can more easily understand the runtime behavior of a program and tune its performance [Du et al. 2011]. HPCs provide access to detailed performance information with much lower overhead than software profilers. Further, no source code modification is needed. The hardware events that can be counted vary from one processor model to another. So does the number of available counters. For example, Intel Pentium III, AMD Opteron and Intel Pentium IV have 2, 4, and 18 hardware counters, respectively. HPC-based profiling tools are currently built into almost every popular operating system (OS) [Intel Inc 2010; Levon and Elie 2010]. Linux Perf [Linux 2010] is a new implementation of performance counter support for Linux. It is based on the Linux kernel subsystem Perf_event, which has been built into 2.6+ systems. The user space Perf tool interacts with the kernel Perf_event by invoking a system call. It provides users a set of commands to analyze performance and trace data. When running in counting modes, Perf can collect specified hardware events on a per-process, per-CPU, and systemwide basis.

\subsection{Static Checking vs. Dynamic Checking}

2.2.1. Static Checking. The HPC-based static checking configures and enables HPC at the beginning of a software module's execution and collects the HPC values only at the end of the execution. The static checking can minimize the performance overhead in the monitored system. However, only checking the final values of the monitored HPCs after an execution leaves an avenue for attackers to carefully craft their malicious code to fool the detection tool.

Consider the scenario that an advanced malware could try to modify the execution path of a program but keep the total occurrences of monitored hardware events unchanged. Specifically, the malware replaces an original function with its own malicious function that generates the same number of hardware events. The static HPC-based technique only checks the occurrences of events at the end of an execution which gives the attacker opportunities to manipulate the count during the execution. Another 
limitation of the static checking is monitoring programs that run for a relatively long time. For example, without the appropriate security patches applied, many web browsers are vulnerable to attacks or exploits. The attacks can be from "bad" websites or malicious plug-ins. In normal use, after launched, a web browser application will keep running until the user shuts it down. The runtime could vary from several minutes to several hours. The malicious actions may only occur at some points of the entire runtime. In this case, checking the total occurrences of events at the end of the execution cannot provide sufficient evidence indicating if any abnormal thing happens during the execution, because the variations in the running time and operations could result in a significant difference in the occurrences of monitored events.

After detecting an abnormal action, another very important step is to determine the specific type of the malware, especially for malware that is well studied. The more information we know about the malware, the easier we can remove it from the infected program or system and mitigate the risk and damage it brings. However, only having the final values of HPCs from the static checking is sometimes not sufficient to accurately determine the details of the malware. One example can be found from the results in Wang and Karri [2013]: The rootkits Suckit and superkit generate very close HPC final values after maliciously modifying the same system calls.

2.2.2. Dynamic Checking. To overcome the limitations in static checking, dynamic checking with periodic sampling has been proposed. Specifically, rather than checking the HPC values only at the end of an execution, we sample the HPCs and get the occurrences of monitored hardware events periodically during the execution. What has been recorded is not a single number of total events counted, but a curve showing how the occurrences of hardware events vary from the beginning to the end of the monitoring period. Two types of malware that have close occurrences of hardware events in a certain monitoring period could have very different shapes in their HPC profiles. The richer specification from the event curve gives more details about the execution of the monitored program, thus significantly increases the detection and identification capabilities. Dynamic checking can also help with monitoring the long running applications discussed above. The HPC profile dynamically generated along the run time is able to expose attacks from normal operations. For example, an installation of a malicious plug-in may generate a very different profile from normal browsing and downloading. By matching the profile with the HPC-based behavior signature in the database, the unintended installation will be detected. The signature could be extracted from the shape of each event curve or the correlation among multiple event curves.

Unlike the static checking which only performs one-time comparison of HPC values at the end of executions, the dynamic checking is based on the analysis of a large number of samples collected. The more HPC samples are analyzed in the dynamic checking, the higher overhead will be introduced. A more accurate detection and identification may require sophisticated classifiers based on various machine learning algorithms such as k-Nearest Neighbors, Artificial Neural Networks, or Decision Tree. Training the classifiers and testing the large number of HPC samples are very compute-intensive tasks [Asha et al. 2013; Ali and Smith 2006], which causes nonnegligible performance overhead.

\subsection{Anomaly-Based Checking vs. Signature-Based Checking}

2.3.1. Anomaly-Based Checking. An anomaly-based check usually has two phases: a training (learning) phase and a detection (monitoring) phase, as shown in Figure 1(a). In the offline training phase, a clean copy of a monitored software module is executed on the platform with a certain HPC configuration. The corresponding HPC values are collected and stored as the "baseline." At runtime, the same HPC configuration 


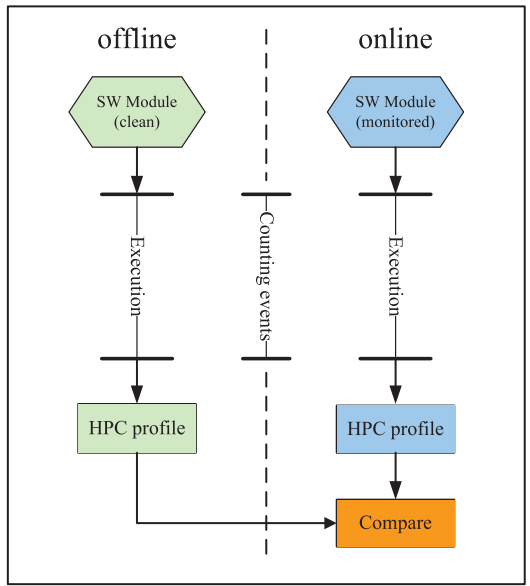

(a)

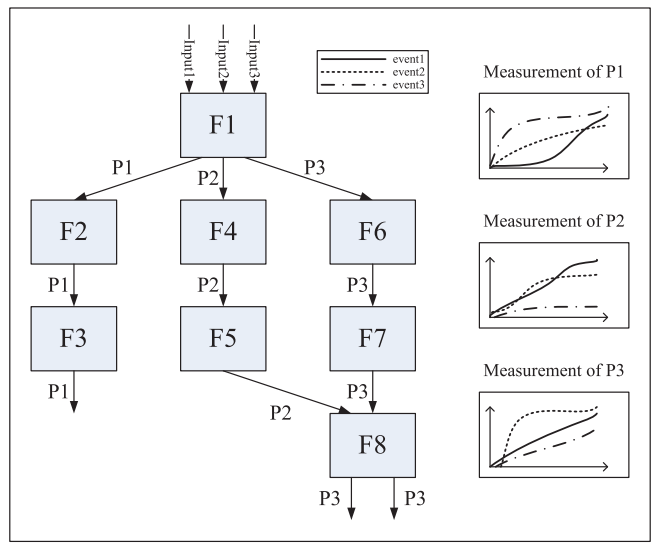

(b)

Fig. 1. (a) The flow of an anomaly-based checking. The HPC values of a monitored software module are compared with the ones of the corresponding clean module. (b) A program with multiple computation paths. The certain computation path is determined by a specific input applied.

is applied to the platform when the monitored module is executed. The HPC values from the online monitoring phase are compared with those from the offline phase. A malicious modification will be suggested if there is an apparent difference between the runtime HPC values and the baseline.

To reduce the false positive, the input to the monitored software module should be specified. A program generally has multiple computational paths in its control-flow graph. Some programs have an infinite number of paths due to loops [Ball and Larus 2000]. Each computation path goes through different functions (or phases) and therefore executes different code. The executions generate different vectors in terms of the occurrences of monitored hardware events, as shown in Figure 1(b). A computational path is primarily determined by the input applied to the program.

2.3.2. Signature-Based Checking. A signature-based checking attempts to model the malicious behavior of malware and uses this model to identify malware. The identification is based on the observation described in Demme et al. [2013]. Regardless of how malware writers change their software, its semantics do not change significantly, and to accomplish a particular task there exist subtasks that cannot be radically modified. It could be expected that they work through a similar set of program phases [Sherwood et al. 2003]. These phases, or trends in the execution, exhibit similar detectable properties in terms of hardware events. This invariance establishes the foundation of the Signature-based dynamic checking.

Figure 2 exhibits the HPC measurements over time for 10 applications from cBench [2010]. The measurement of each application consists of four hardware event series. The length of the measured series and the location and shape of all the ups and falls provide meaningful information that can be used to differentiate one operation from another, making the HPC measurement a unique signature of a particular behavior of an application.

\subsection{Virtualization-Based Checking}

For the malware which has the access to the kernel space of a system, such as a kernel rootkit, the HPC-based detection facility should be deployed somewhere that the privileged attacker cannot reach. One of the solutions is using virtualization 

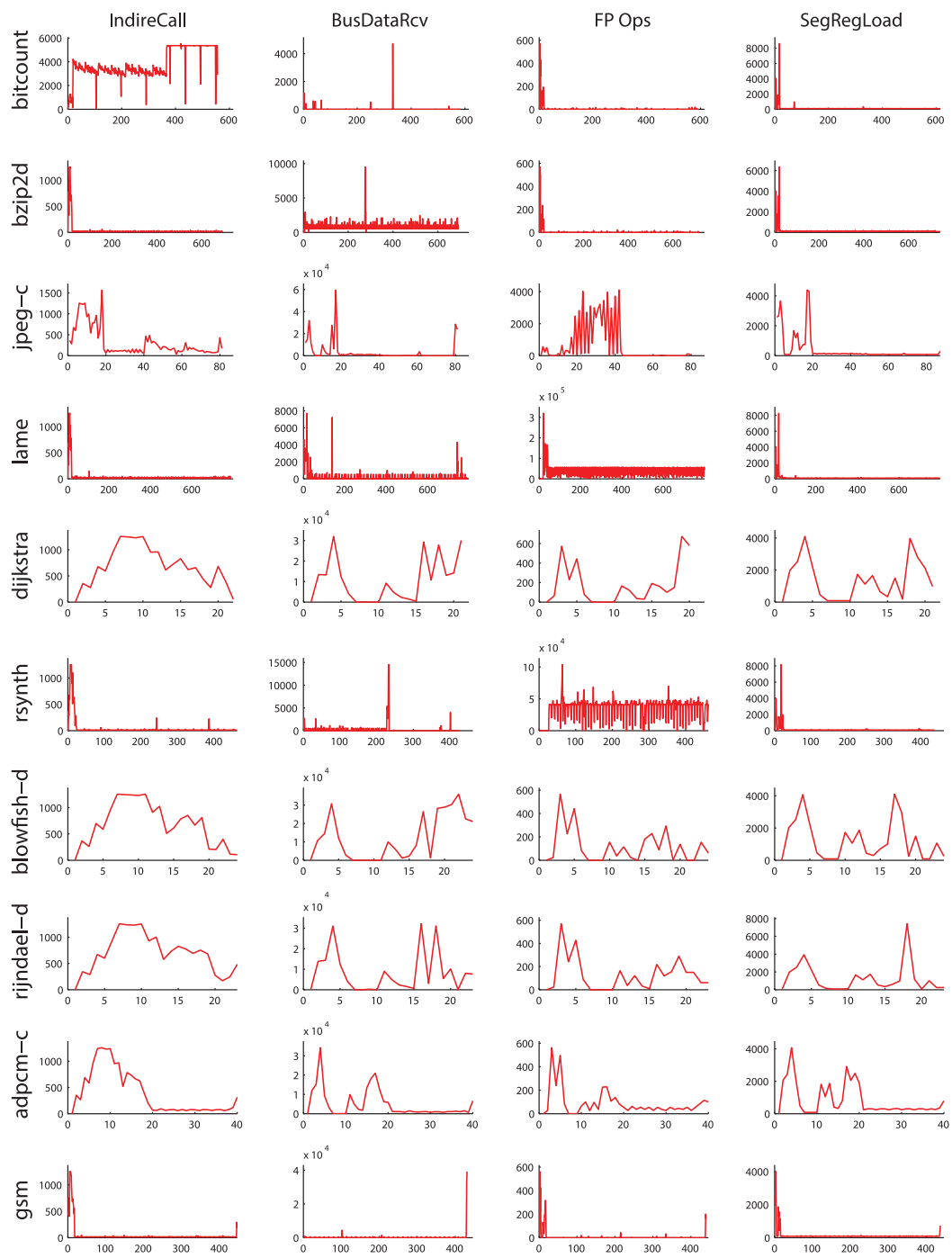

Fig. 2. Performance counter measurements over time for 10 applications from cBench. The measurement for each application consists of four hardware event series and apparently differs from other applications'. The measurement can be used as the signature of a particular behavior of an application.

technology. The virtualization system provides extra isolation between the guest OS and the underlying hardware. The HPCs are directly controlled by the trusted Virtual Machine Monitor (VMM) while the compromised guest OS has no access to them. An applicable example is using HPCs to detect kernel rootkits which modify system calls in the OS kernel to hide themselves and perform other malicious actions [Wang and Karri 2013]. In the offline profiling phase, the executions of system calls of the original trusted guest OS are characterized by the HPC-based profiler running in the underlying VMM. The measured results are stored in the VMM as the "clean copy." In the online runtime checking phase, system calls of a running monitored guest OS are measured and compared with that of the corresponding trusted guest OS. If the 


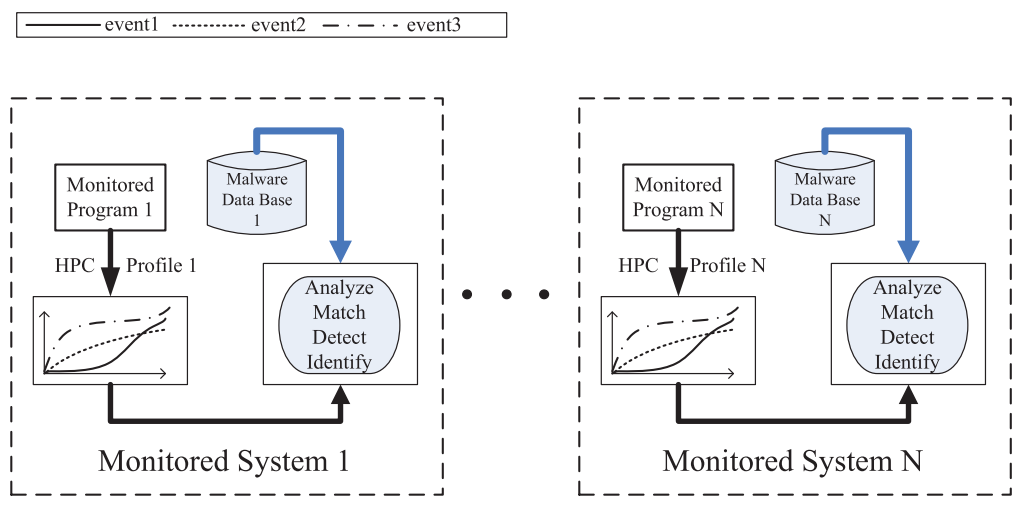

(a)

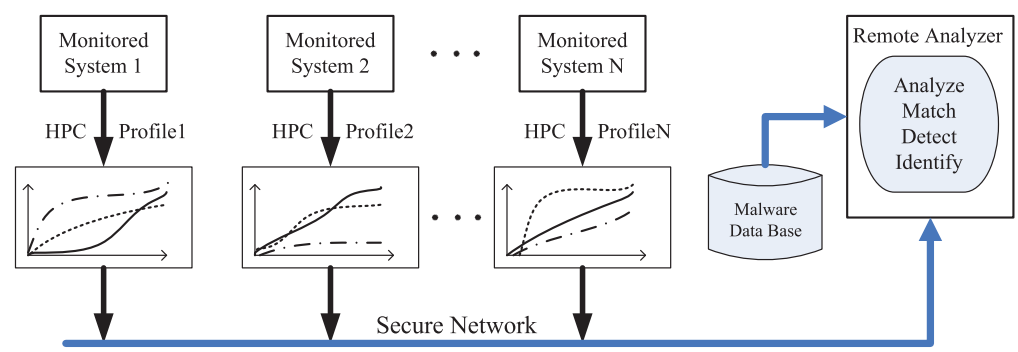

(b)

Fig. 3. (a) The original HPC-based technique which has the malware database and performs the analysis locally. (b) A "sample-locally-analyze-remotely" infrastructure for malware detection and identification. The periodically sampled HPC values are sent to a remote server for analysis.

execution flow of a system call is maliciously modified, then the measured number of monitored hardware events will differ from the uninfected execution.

\section{REMOTE MALWARE DETECTION AND IDENTIFICATION WITH COMPRESSIVE SENSING}

The HPC-based detection and identification techniques usually take two steps: logging the occurrences of the monitored HPC events and performing the analysis on the captured data. Compared to software loggers, HPCs provide low-overhead access to key performance indicators related to a processor's functional units, caches, and main memory since they are running automatically in the hardware. In the logging step, detailed information is obtained with minimal impact on system performance.

However, as mentioned in Section 1, analyzing the captured data (specifically, performing the matching and classification on the data) may introduce significant storage overhead to the monitored system. Assume in a smart grid there are $N$ nodes running critical software that needs to be monitored, as shown in Figure 3(a). Each node runs the same software modules with the same hardware configuration (e.g., power meters in the customer premise). With the HPC-based malware detection and identification enabled, $N$ local databases are required for the whole smart grid while each of them has to contain the complete set of HPC profiles of the corresponding clean software modules and existing known malware. Each database is actually a duplicate of another. Besides the storage overhead, performing the matching and classification is also a compute-intensive task which consumes a lot of computational resources in the monitored system. 
Fortunately, the storage and compute-intensive analysis is not necessary to be run locally in the monitored system. After the monitored HPC values are collected, the following step of the HPC-based checking does not depend on the state of the monitored system. One effective way to reduce the consumption of local resources is to perform all the complex analysis in a remote server with much more storage and computing capabilities. Figure 3(b) shows the infrastructure we propose that provides a "samplelocally-analyze-remotely" check. Specifically, the HPC profiles containing the behavior records of the monitored programs are captured periodically from local systems. Then the data are sent through a dedicated secure network to a remote server for further detection and identification. With this infrastructure, only one database connected to the remote server is required for the $N$ monitored systems that run the same software modules. There is no extra storage needed at each monitored platform and the storage overhead of the whole system is reduced by $N$ times. This infrastructure also minimizes the local performance overhead because the matching and classification algorithms are run remotely.

The proposed infrastructure brings the benefit of storage and performance overhead reduction. However, it increases the I/O bandwidth requirement. Based on the experimental results we obtained from running a set of Linux applications, we observed that for some particular applications, to collect sufficient information to differentiate the normal operation of an application from an operation of another application or an expected operation of malware, the granularity of the sampling should be smaller than 100,000 instructions. Specifically, at lease one sampling on the monitored events should be taken when every 100,000 instructions are executed. When applying such a granularity to the sampling on a general computing platform (e.g., Intel Core CPU), approximately 25,000 samples will be generated per second. With four bytes per sample and 10 events monitored simultaneously, there is a raw measurement bandwidth requirement of $1 \mathrm{MB} / \mathrm{s}$ for each monitored platform. It may be further increased when higher detection/identification accuracy is required. On the remote server side, the I/O bandwidth problem is even more critical, especially when many monitored platforms are sending out the data at the same time.

To overcome the I/O bottleneck of using fine-grained sampling of the HPC profiles for remote malware detection and identification, we propose an approach that creates a compressed version of the measurement vector (e.g., $5 \times$ smaller than the original sampled data) and writes it out for remote analysis. We demonstrate that highly accurate malware detection and identification can be performed from far fewer measurements than the total number of samples. Reducing the number of measurements beneficially lowers the portion of monitoring bandwidth devoted to monitoring data, solving the "monitoring data deluge" problem. The mathematical framework that allows accurate sensor data recovery from highly incomplete measurements is called Compressive Sensing and forms a key element of this work. Recent advancements in Compressive Sensing algorithms allow accurate recovery of the fine-grained HPC profiles from their compressed measurements. Also an adaptive sensing is incorporated into the monitoring system that, over time and in a dynamic fashion, determines and optimizes the utility of each sensing type to overall reliability. Because the Compressive Sensing data are a highly accurate representation of the original sensor data, it can be used with confidence in malware detection/identification and feedback control mechanisms (e.g., dynamic sampling rate adjustment).

\subsection{Background of Compressive Sensing}

Compressive Sensing (also known as compressed sensing, compressive sampling, or sparse sampling) is a signal processing technique for efficiently acquiring and reconstructing a signal by finding solutions to underdetermined linear systems. This takes 


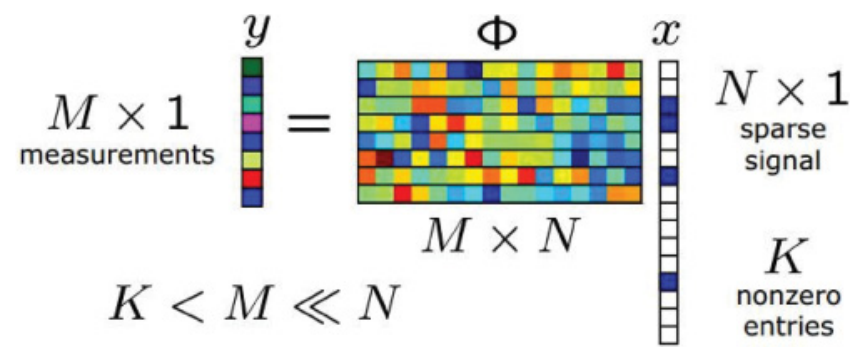

Fig. 4. Compressive Sensing creates a small number of linear measurements from a larger, but unknown, data vector $\mathbf{x}$. A decoder, not shown, recovers an estimate of $\mathbf{x}$ from $\mathbf{y}$ using a nonlinear algorithm. In order to produce an accurate estimate, the unknown signal $\mathbf{x}$ must be sparse in some domain. In a data center application, $\mathbf{x}$ contains raw sensor measurements at some time instant; $\mathbf{y}$ is a compressed version of $\mathbf{x}$ that beneficially uses less network bandwidth. A node running special software reconstructs a highly accurate estimate of $\mathbf{x}$ from the incomplete measurements $\mathbf{y}$.

advantage of the signal's sparseness or compressibility in some domain, allowing the entire signal to be determined from relatively few measurements.

Figure 4 is a pictorial overview of Compressive Sensing. An unknown signal $\mathbf{x}$ is modeled as a column vector with $N$ components. A second column vector $\mathbf{y}$, called the measurement vector, is produced from $\mathbf{x}$ through multiplication by an $M \times N$ sensing matrix $\Phi$. In our application, $\mathbf{x}$ is the underlying collection of $N$ HPC measurements; $\mathbf{y}$ is the compressed version of this data, containing $M$ measurements. Mathematically, this can be expressed by the following matrix equation:

$$
\mathbf{y}=\Phi \mathbf{x}
$$

We would like to recover the unknown vector $\mathbf{x}$ from the measured vector $\mathbf{y}$. We would also like the number of measurements, $M$, to be less than $N$ so we can achieve bandwidth savings in our computing system. When $M<N$, the equation above is underdetermined, and an infinite number of solutions exist in the general case. However, if the vector $\mathbf{x}$ is sparse in some domain, that is, $\mathbf{x}$ has no more than $K$ nonzero coefficients either in its native sampling domain or in some transformed domain, then $\mathbf{x}$ can be accurately recovered using one of several nonlinear optimization algorithms, such as basis pursuit or matching pursuit [Eldar and Kutyniok 2012].

\section{IMPLEMENTATION DETAILS}

This section describes the implementation details of our proposed technique. To apply the Compressive Sensing technology to the HPC-based technique, the following challenges need to be carefully considered:

-Reducing the noise of the periodic sampling. The periodic sampling can be performed in different ways. One of the ways is letting a counter keep counting from the beginning to the end. Samples are taken periodically over the course of the duration. Every sampled number is the accumulation of all the previous ones. When access to a counter is made, some noise occurs (for example, from handling the HPC interrupts). The noise will be counted into every sampled number if the counter keeps counting. Though the noise in each sampling is relatively small, it will propagate during the monitoring period if the event count is accumulated by every sampling. In this situation, the obtained HPC profile will distinctly deviate from the actual fine-grained profile, especially when the monitoring period is long. To avoid a propagated noise, we reset the counter for every sampling to perform a "differentiated sampling." That is, a sampled number is the event count only from the current sampling period rather than an accumulated sum. By doing this, the events 
generated from the execution of any code out of the monitored program will not be counted so the noise will be significantly reduced. In this way, the actual captured HPC profile is the differentiation of the accumulated one.

Another disturbance is from the sampling interval. The intuitive interval can be a certain amount of time, for example, every millisecond. However, because the execution time for the whole program or a block of code has much variation when the system state changes from time to time, the HPC measurement between the same two time-based check points of a given program will most likely be inconsistent from one execution to another. In our implementation, we use the occurrences of retired instructions as the checking interval for measuring an execution. The correlation between the occurrences of retired instructions and the occurrences of other monitored hardware events is relatively strong, making the measurements more reliable.

-Determination of sparsifying function. Compressive Sensing assumes that the signal to be recovered is sparse in some domain. In other words, it should have relatively few nonzero components, or "spikes," in its waveform. In some cases, the original HPC profiles with differentiated sampling may not be sparse enough. Further differentiating the HPC profiles $n$ times (i.e., taking the $n$th derivative of the HPC profile) could increase the sparsity but will also accentuate noiselike patterns in the signal. A challenge will be to determine the best sparsifying function to use and to determine whether a single sparsifying function will work for all malware types. From the initial experiments, we observe that the first derivative $(n=1)$ is a good initial choice, and we start with that as a baseline. A further analysis may require different values of $n$ for the best performance, and then the optimal value will be part of the metadata that gets associated with that malware type in the database.

-Determination of optimal sensing matrix $\Phi$. In order for the compressed measurement vector $\mathbf{y}$ to capture all of the relevant information in the sparse vector, the sensing matrix $\Phi$ must exhibit certain properties, such as the Restricted Isometry Property [Candès 2008]. In practice, it has been found that random Gaussian or Bernoulli matrices have such properties, but there are still tradeoffs between the computational complexity of forming a measurement and the minimum number of measurements that are required for accurate recovery. We work with Bernoulli matrices having entries of $0,+1$, or -1 . Forming a measurement vector with a Bernoulli matrix has the beneficial property of only requiring additions and subtractions, but slightly more measurements are required compared to Gaussian matrices. For best results, this matrix must be codesigned with the sparsifying function. If the sparsifying function produces at most $\mathrm{K}$ spikes, and if there are $\mathrm{N}$ fine-grained samples, then $M$, the minimum number of measurements, must satisfy the following inequality: $M \geq C \log (N / K)$, where $C$ is a small constant, like $C=5$. The challenge is to design an $M \times N$ sensing matrix $\Phi$ with $M$ as small as possible, that is, computationally efficient and that provides accurate recovery of a K-sparse signal using existing Compressive Sensing recovery algorithms.

-Determination of optimal Compressive Sensing decoding algorithm. Over the past decade, numerous algorithms have been described and implemented for Compressive Sensing decoding [Gorodnitsky and Rao 1997; Tropp and Gilbert 2007; Willett et al. 2010]. Algorithms differ in terms of speed, performance, and amount of fine-tuning that is required. We start with efficient Compressive Sensing recovery algorithms having good performance and intuitive tuning parameters (e.g., TVAL3 [Li et al. 2009]). In some cases when results are subpar, more advanced algorithms (e.g., Fast Bayesian Matching Pursuit [Schniter et al. 2008]) could be applied but may have to sacrifice speed for enhanced performance.

-Robust profile matching for malware identification. Once a profile is recovered, it must be matched against a database of malware profiles that has been previously compiled under controlled conditions. The challenge is to determine when a profile is 


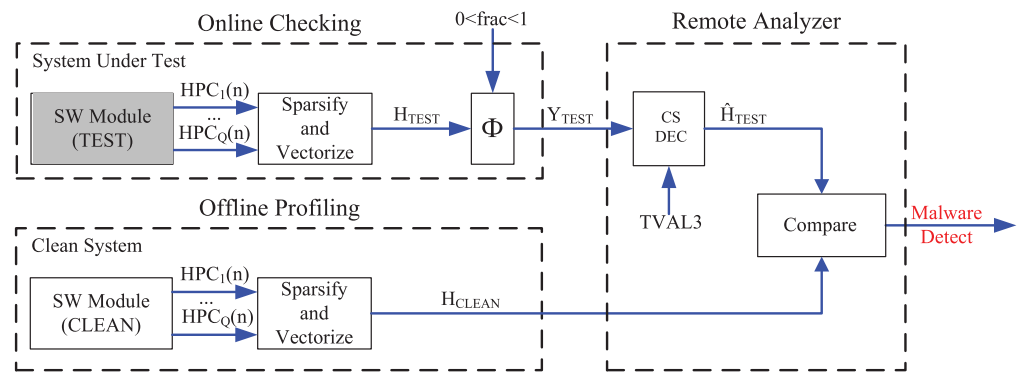

(a)

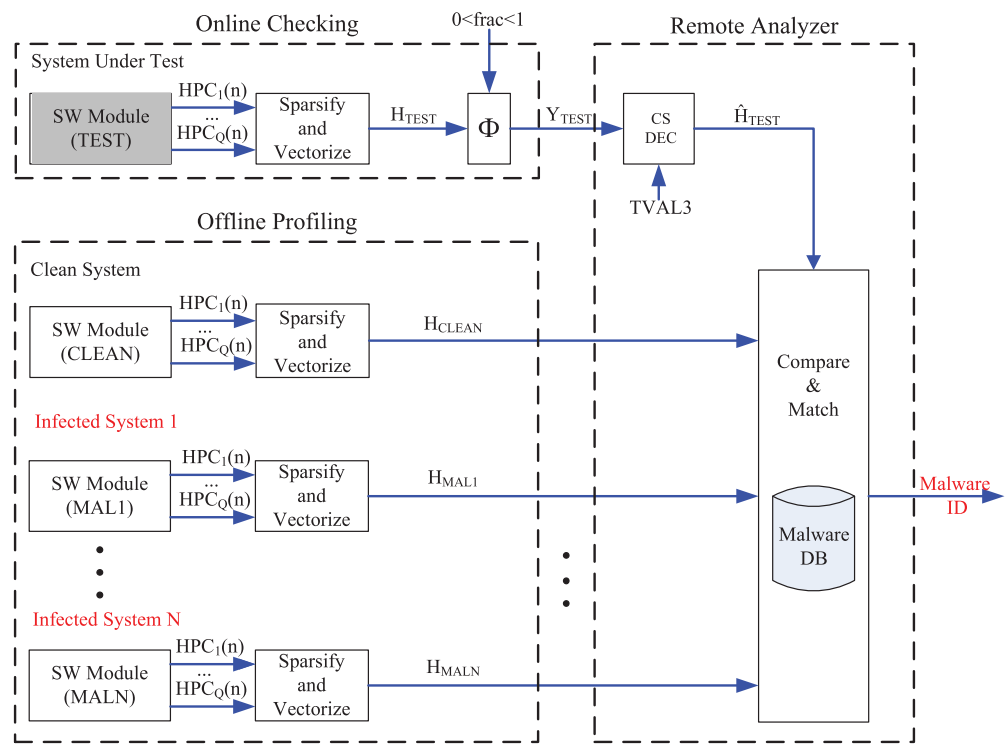

(b)

Fig. 5. Malware detection architecture (a) and identification architecture (b).

close enough to that of a known profile or whether it represents a new, uncategorized type of malware. In our proof-of-concept experiment, we simply use Pearson's correlation coefficient [Loğoğlu and Ateş 2010] to measure the similarity between the tested profile and the reference profile. For a more comprehensive analysis, the classification algorithms, such as k-means clustering [Kanungo et al. 2002] and adaptive boosting [Lin 2008], can be applied.

\subsection{Detection/Identification Architecture}

The technical details of the detection architecture is described by referencing the block diagram shown in Figure 5(a). At the monitored platform, a software module under test is launched with a known set of inputs. HPCs are collected over the course of the module's duration. The HPC samples collected from the differentiated sampling are first sparsified and vectorized before being compressed into a much smaller vector, $Y_{T E S T}$, by a linear measurement matrix $\Phi$, with a proper compression ratio. The compressed profile will be sent out through a secure network to the remote analyzer for analysis.

On the remote analyzer side, the received compressed profile of the under-test module is first recovered to an estimate of the fine-grained profile using the sophisticated, 
nonlinear Compressive Sensing recovery algorithm TVAL3. The recovered profile is then compared with the "golden" HPC profile stored in a database. The "golden" copy is generated offline from the execution of the same software module but in a clean system. The Pearson's correlation coefficient is measured to determine if the software module under test is maliciously modified. If the calculated Pearson's correlation coefficient is smaller than a preset noise threshold, then a malicious modification to the software module under test is suggested.

If the software module under test is considered to be compromised, then the identification process, shown in Figure 5(b), will be run to perform further analysis to determine if the anomalies are caused by any known type of malware. The identification is performed by matching the recovered profiles with the HPC-based malware signatures stored in the database. These signatures are collected from offline evaluations of malicious actions that have already been observed and studied. The database keeps getting updated when new malware activities are found. For the current implementation, the matching is based on the Pearson's correlation coefficient between the detected malware profile and the malware signatures in the database. Sophisticated classifiers based on machine learning algorithms may be required to further determine which type of malware it belongs to. If the profile under test matches one of the known malware signatures, which means it has been successfully identified, the corresponding actions will be taken in the monitored system to remove the malware or reduce the damage it may cause. If the profile under test does not match any signature in the database, then there are two possible situations: (1) the malware is new and has not been recorded in the database or (2) the accuracy of the recovery is not high enough. Our technique will first increase the compression ratio to obtain a more accurate recovered HPC profile and repeat the matching procedure. If there is still no match found even though the highest compression ratio is reached, that is, the profiles without compression are checked, then an unknown type of malware will be reported.

\section{RESULTS AND ANALYSIS}

In this section, we perform several experiments to compare the effectiveness of Compressive Sensing and conventional sampling, evaluate the capabilities of malware detection and identification with Compressive Sensing, and analyze the performance overhead in the monitored system.

\subsection{Compressive Sensing vs. Conventional Sampling}

An accumulation-style fine-grained HPC profile of a program exhibits ramp-ups that may be slow or fast. If the program is modified or a piece of malicious code is inserted, then ramp-ups with different gradients will be introduced to the original HPC profile. Figure 6 shows a simple example. The execution of the malicious code generates a "jump" (pointed by the arrow in Figure 6(a)) in the fine-grained HPC profile. With the "differentiated sampling" described before, the ramp-ups become steps with different heights in the actual captured profile, presented in Figure 6(b). In this example, a total of 250 samples are collected. Because the duration of the malicious action is relatively short compared with the whole monitoring period, the small "jump" turns to a "spike."

An effective detection or identification relies on all the "spikes" that appear in the profile waveform. However, due to the I/O limitation, only a reduced version of the collected data can be sent out for analysis. If the conventional sampling at regular intervals is used to reduce the data size, then these "spikes" may be missed if the sampling rate is low. Figure 6(c) shows an example. Assume that the I/O bandwidth only allows $25 \%$ of the 250 HPC samples to be sent out for analysis. If we run conventional sampling with the equal intervals, that is, one of every four samples is sent out, then 


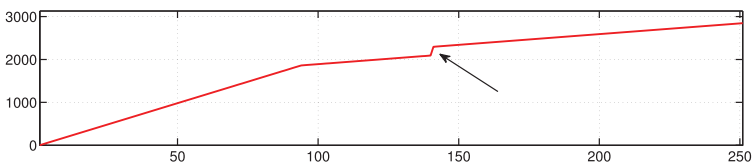

(a)

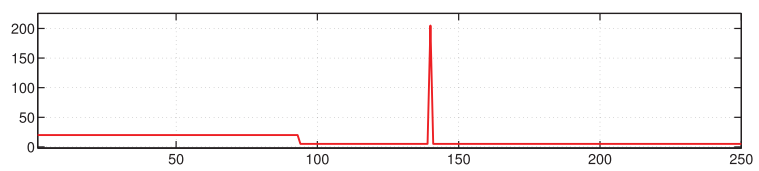

(b)

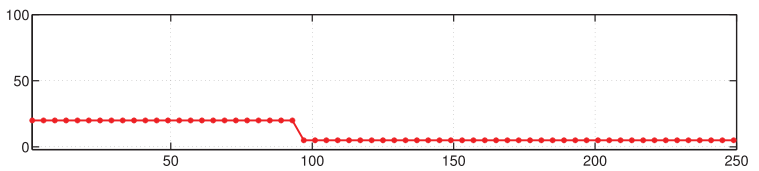

(c)

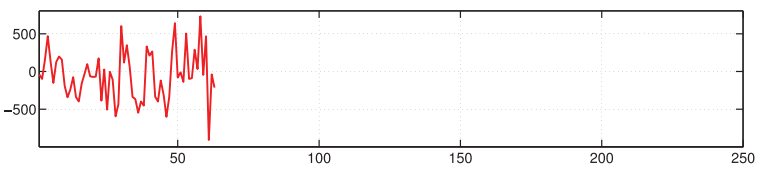

(d)

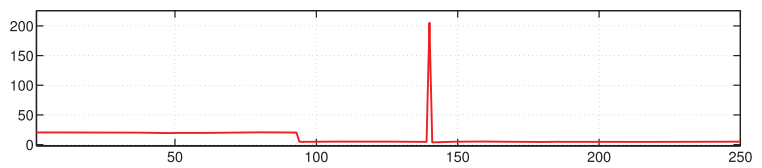

(e)

Fig. 6. The accumulation-style HPC profile with a "jump" in the middle (a). The original sampled HPC profile (b). The reduced HPC profile after conventional sampling (c). HPC profile after compression with Compressive Sensing. The size is $25 \%$ of the original profile (d). Profile after Compressive Sensing recovery (e).

the "spike" is not observed after the sampling. The probability of detecting the "spike" is only $25 \%$.

With Compressive Sensing and the corresponding recovery algorithm, these "spikes" can be $100 \%$ detected with the same reduced data size. By applying a sensing matrix, the original profile is compressed into a reduced version with a length of $25 \%$, shown in Figure 6(d). The reduced samples are sent out and then accurately recovered to an estimated full profile, shown in Figure 6(e).

\subsection{Choosing Proper Events}

Recent models of mainstream processors have hundreds of architectural/ microarchitectural hardware events available for monitoring. The hardware events on a platform can be categorized into different groups, such as load/store events, cache events, execution unit events, crossbar events, and so on. This gives us many options to model a HPC-based signature. However, not all the available hardware events are good candidates for modeling a recognizable signature, because the occurrence of some events depends on the current system state, which constantly changes. For such events, 

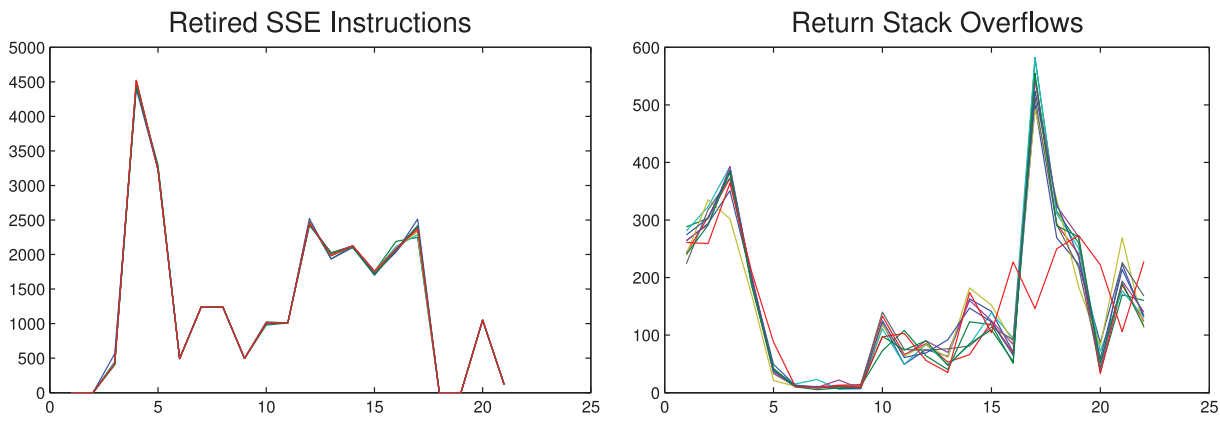

ES Segment Register Loads
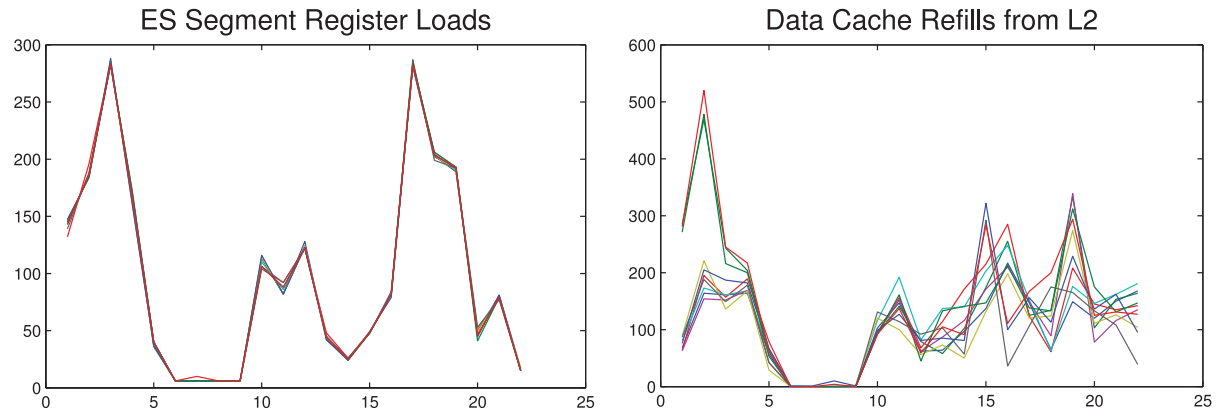

Fig. 7. Measurements of different hardware events over time on the stringsearch benchmark. The number of retired SSE instructions and ES segment register loads are both more consistent than return stack overflows and data cache refills from $L 2$ over the repeated execution.

the number of occurrences during execution vary dramatically from one execution to another when the total workload of the system changes or when certain applications are running simultaneously. To allow for a signature to be extracted properly, the signature must be repeatable and robust. It is important to carefully select proper hardware events whose occurrences are more consistent every time when the targeted application is executed.

One example is shown in Figure 7. The occurrences of retired SSE instructions, ES segment register loads, return stack overflows, and data cache refills from L2 are measured respectively during the execution of the same stringsearch benchmark which is repeated 5 times. Different workloads are applied to the measured system. We can see from the results that for retired SSE instructions and ES segment register loads, the curves for different executions are very coincident despite system state changes. The signature based on those hardware events should therefore be robust to noise and easy to retrieved in a repeatable manner. In contrast, the measurements for return stack overflows and data cache refills from L2 have very significant deviations when the execution environment varies.

To determine which hardware events are more robust for modeling an application's fingerprint, we perform the experiments on a platform with a 2.3-GHz AMD Quad-Core Opteron 1356 CPU with 8GB RAM. The operating system is 32-bit Ubuntu 12.04 (kernel version 3.0.16). All the available hardware events are evaluated by executing the same set of benchmarks from cBench multiple times with varying system workloads. A tool called Stress [2010] is used to impose a configurable amount of CPU, memory, and I/O workloads on the system. The repeatability is quantified with the Coefficient of Variation (C.V). Table I lists the hardware events with smallest C.Vs. The results exhibit that most of the robust events are from the execution unit. 
Table I. Coefficient of Variation (\%) of Different Hardware Events, Retired SSE Instructions (SSE), Multiply Ops (MUL), Fastpath Double Op Instructions (FPD), ES Segment Register Loads (ESS), Retired Near Returns (RET), Retired Uops (UOP), Retired Branch Instructions (BRN), and Retired Taken Branches (BRT), Measured from the Execution of 10 Applications, Bitcount (bi), bzip2d (bz), jpeg_c (jp_c), Lame (la), Dijkstra (di), Rsynth $(r s)$, Stringsearch (st), Blowfish_d (bl_d), adpcm_d (ad_d) and gsm (gs), with Varying System Workloads. These Events Have Smaller C.Vs, Indicating a Better Repeatability. Therefore, They Are the Good Candidates for Monitoring. The Numbers Are Calculated Over 10 Repeated Runs for Each Application

\begin{tabular}{|c|c|c|c|c|c|c|c|c|c|c|c|}
\hline \multirow{2}{*}{$\begin{array}{l}\text { Event } \\
\text { name }\end{array}$} & \multicolumn{10}{|c|}{ Application } & \multirow{2}{*}{$\begin{array}{l}\text { Average } \\
\text { C.V }(\%)\end{array}$} \\
\hline & bi & $\mathrm{bz}$ & jp_c & la & di & rs & st & bl_d & ad_d & gs & \\
\hline SSE & 0.01 & 0.02 & 0.02 & 0.03 & 0.02 & 0.02 & 0.02 & 0.02 & 0.02 & 0.02 & 0.02 \\
\hline MUL & 0.71 & 0.44 & 0.46 & 0.00 & 0.41 & 0.00 & 0.32 & 0.24 & 0.44 & 0.37 & 0.34 \\
\hline FPD & 0.41 & 0.58 & 0.65 & 0.23 & 0.58 & 1.02 & 0.70 & 0.48 & 0.57 & 0.37 & 0.56 \\
\hline ESS & 1.70 & 1.29 & 0.98 & 0.56 & 0.94 & 0.41 & 0.20 & 0.20 & 0.35 & 1.02 & 0.76 \\
\hline RET & 0.09 & 2.58 & 0.22 & 3.06 & 6.72 & 0.02 & 0.36 & 0.19 & 0.99 & 0.98 & 1.52 \\
\hline UOP & 1.95 & 2.09 & 2.76 & 2.39 & 1.87 & 2.22 & 1.44 & 1.21 & 2.22 & 1.45 & 1.96 \\
\hline BRN & 2.98 & 2.09 & 1.55 & 2.37 & 2.01 & 1.67 & 2.11 & 2.21 & 2.26 & 1.55 & 2.08 \\
\hline BRT & 1.55 & 2.17 & 3.29 & 1.99 & 2.65 & 2.17 & 2.51 & 2.45 & 2.42 & 2.30 & 2.35 \\
\hline \multicolumn{11}{|c|}{ Average over all tested events $(120+)$} & 49.7 \\
\hline
\end{tabular}

Table II. The Pearsons Correlation Coefficient Between a Recovered HPC Profile and the Original Profile for Each of the 10 Applications. The Numbers Are Averaged Over Four Hardware Events, SSE, MUL, FPD, and ESS, Selected from Table I

\begin{tabular}{|c|c|c|c|c|}
\hline \multirow{2}{*}{ Application } & \multicolumn{4}{|c|}{ Average Pearson's correlation coefficient } \\
\cline { 2 - 5 } & $\mathrm{CR}=0.4$ & $\mathrm{CR}=0.3$ & $\mathrm{CR}=0.2$ & $\mathrm{CR}=0.1$ \\
\hline \hline bitcount & 0.92 & 0.89 & 0.80 & 0.57 \\
\hline bzip2d & 0.95 & 0.90 & 0.82 & 0.60 \\
\hline jpeg_c & 0.89 & 0.90 & 0.80 & 0.55 \\
\hline lame & 0.94 & 0.87 & 0.81 & 0.66 \\
\hline dijkstra & 0.96 & 0.91 & 0.85 & 0.65 \\
\hline rsynth & 0.87 & 0.83 & 0.77 & 0.52 \\
\hline stringsearch & 0.88 & 0.85 & 0.75 & 0.49 \\
\hline blowfish_d & 0.95 & 0.90 & 0.82 & 0.63 \\
\hline adpcm_d & 0.92 & 0.87 & 0.79 & 0.63 \\
\hline gsm & 0.91 & 0.87 & 0.80 & 0.62 \\
\hline \hline
\end{tabular}

To demonstrate that the HPC profiles built from selected repeatable events can be successfully recovered from the compressed version, the four most robust hardware events, SSE, MUL, FPD and ESS, selected from Table I are periodically sampled (one sampling every 100,000 instructions) during the executions of the 10 tested applications. The obtained profiles are compressed by Compressive Sensing and then recovered with the recovering algorithm TVAL3. Different compression ratios from 0.1 to 0.4 are applied. The recovered profiles are compared with the original clean HPC profile without Compressive Sensing. The quantified similarities of the original and recovered profiles are presented in Table II. For each application the Pearson's correlation coefficient between a recovered HPC profile and the original profile is calculated. The range is from -1 to 1 (in this experiment, 1 means the best match). The coefficients in the table are averaged over the four monitored hardware events.

\subsection{Malware Detection with Compressive Sensing}

In this experiment, we demonstrate that a recovered HPC profile after Compressive Sensing can still provide accurate information for detecting malicious modification to a program. We take kernel rootkit as an example. As the main purpose of a kernel rootkit is to hide malicious processes, files, connections, and so on, a common action 


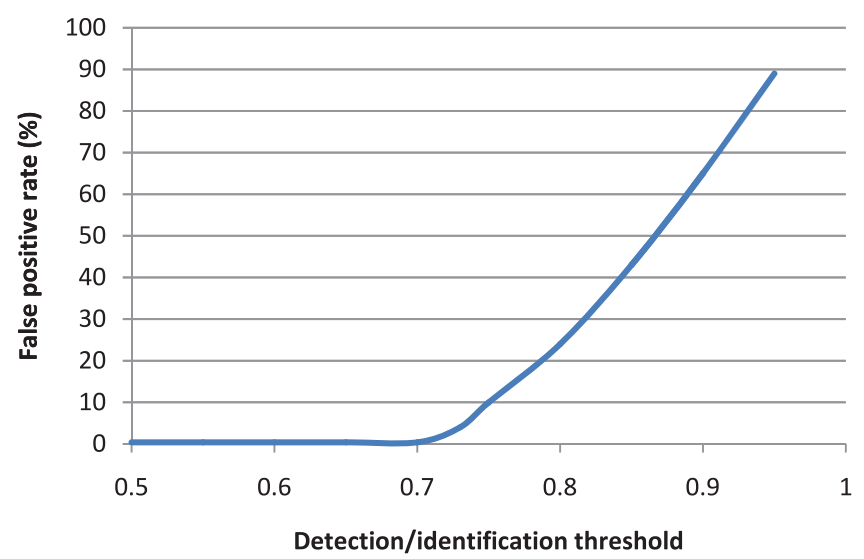

Fig. 8. False positive rate with different thresholds of the Pearson's correlation coefficient.

that a kernel rootkit performs is to fool the user monitoring utilities (such as $p s, l s$, netstat in Linux). These monitoring utilities retrieve the information about the system states by invoking a sequence of system calls. Kernel rootkits usually manipulate the normal execution of system calls to prevent the monitoring utilities from obtaining the correct information. For example, the Linux $p s$ command will return the status of all the running processes. The system calls invoked by the $p s$ command include sys_open, sys_close, sys_read, sys_lseek, sys_stat64, sys_fstat64, sys_getdents64, sys_old_mmap, and so on. A rootkit modifies these system calls so the information about the malicious processes will not appear in the list returned by $p s$. The work presented in Wang and Karri [2013] shows that the modifications usually result in a significantly different number of monitored hardware events from the uninfected execution (at least 5\%; for some rootkits, the deviation can be even larger than $1000 \%$ ).

We select four robust hardware events, retired near returns (RET), retired uops (UOP), retired branch instructions (BRN), and retired taken branches (BRT), from Table I (some events with better repeatability such as SSE and MUL do not exhibit enough occurrences during the execution of the tested system calls and thus are not selected). As the example, we perform the experiment on the system call sys_open() since it is the common target for many known kernel rootkits. We obtain the HPC profiles from the executions of sys_open() manipulated by a set of different real-world kernel rootkits, SucKIT 1.3b, Sk2rc2, Superkit, Adore 0.42, Phalanx b6, Sebek 3.2, Adore-ng, and KBeast.

Considering the slight disturbance from the changing system state, it can be expected that the HPC profile of a clean system call could slightly differ from one execution to another. One situation we must consider is false positives. To determine the threshold of the Pearson's correlation coefficient for detecting and identifying malware, we first run the experiment multiple times on the clean sys_open(). The Pearson's correlation coefficients between a recovered HPC profile of the clean sys_open() and the original uncompressed profile are calculated. Figure 8 shows the false-positive rate when different noise thresholds are applied. From the results, we can observe that the false-positive rate becomes 0 when the threshold of the Pearson's correlation coefficient is less than 0.70 . So we can safely set the detection and identification threshold to 0.70 . That is, if the coefficient between a profile under test and the original clean profile is less than 0.70 , then a malicious modification is suggested.

We then perform the experiments on the system call modified by different rootkits. The Pearson's correlation coefficients between a recovered HPC profile of sys_open() 
Table III. The Pearsons Correlation Coefficient Between a Recovered HPC Profile of sys_open() Modified by a Kernel Rootkit with Different Compression Ratios and the Original Uncompressed Profile

\begin{tabular}{|c|c|c|c|c|}
\hline \hline \multirow{2}{*}{ Application } & \multicolumn{4}{|c|}{ Average Pearson's correlation coefficient } \\
\cline { 2 - 5 } & CR $=0.4$ & CR $=0.3$ & CR $=0.2$ & CR $=0.1$ \\
\hline \hline SucKIT 1.3b & 0.01 & 0.01 & 0.02 & -0.02 \\
\hline Adore 0.42 & 0.04 & 0.05 & 0.04 & 0.02 \\
\hline Sk2rc2 & 0.04 & 0.07 & -0.01 & 0.01 \\
\hline Superkit & 0.05 & 0.08 & 0.05 & 0.06 \\
\hline Phalanx b6 & 0.43 & 0.50 & 0.35 & 0.42 \\
\hline Sebek 3.2 & 0.35 & 0.40 & 0.33 & 0.38 \\
\hline Adore-ng & 0.60 & 0.56 & 0.47 & 0.49 \\
\hline KBeast & 0.21 & 0.25 & 0.33 & 0.30 \\
\hline clean & 0.95 & 0.88 & 0.80 & 0.62 \\
\hline \hline
\end{tabular}

modified by a kernel rootkit with different compression ratios and the original uncompressed profile are presented in Table III. The correlation coefficients between a recovered HPC profile of the clean sys_open() and the original profile are also presented. From the results shown in Table III, when the compression ratio is larger than 0.1, both the clean and malicious profiles are successfully classified. No false positive or false negative is encountered. When the compression ratio is 0.1 , the recovered clean profiles are classified as malicious due to a low average correlation coefficient.

\subsection{Malware Identification with Compressive Sensing}

The next experiment is to test the identification capability of the Compressive Sensingbased technique. Since each rootkit modifies sys_open() in its own way, the shapes of the HPC profiles captured will differ, which can be used as the behavior signatures for the rootkits. To demonstrate the effectiveness of the recovery-based malware identification, the recorded profiles are compressed, recovered, and then compared with the original uncompressed profiles. We obtain the HPC profiles from the executions of sys_open() manipulated by the same kernel rootkits mentioned above. And the same hardware events, RET, UOP, BRN, and BRT, are monitored. We take SucKIT 1.3b and Superkit as examples and show the details in Figure 9.

Figure 9(a) shows the original BRN and RET profiles for the rootkits SucKIT 1.3b and Superkit. The $\mathrm{X}$ axis indicates the number of samples while the $\mathrm{Y}$ axis is the derivative of the occurrences of the monitored event. The compressed and recovered profiles with different compression ratios are shown in Figures 9(b), 9(c), 9(d), and 9(e). From the figure, we can observe these profiles are mostly recovered when the compression ratio is 0.2 or larger. In this experiment, again we use the Pearson's correlation coefficient to quantitatively measure the similarity between two HPC profiles. The original rootkit profiles are stored as signatures in the database as references. The compressed profiles to be analyzed are recovered and compared with the signatures in the database. A detected malware $M x$ will be identified as a known rootkit $R a$ if the Pearson's correlation coefficient between $M x$ and $R a$ is the largest, and it is larger than a preset threshold of 0.70. If the Pearson's correlation coefficient between $M x$ and any rootkit reference in the database is smaller than 0.70 , then $M x$ will be reported as an unknown malware.

Table IV presents the Pearson's correlation coefficients between the recovered profiles of all the tested rootkits and the original profiles of reference rootkits in the database. Each number is the average over the experiments on all four hardware events BRN, RET, BRT, and UOP (INS is used for sampling). The results indicate that when the compression ratio is larger than or equal to 0.2 , the average Pearson's 

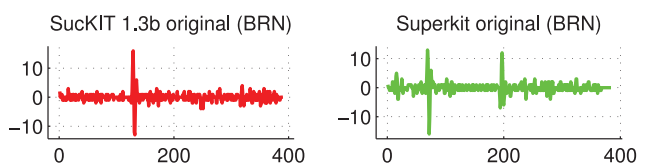

(a)
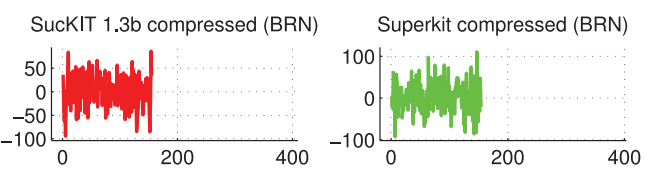

SucKIT 1.3b recovered (BRN)
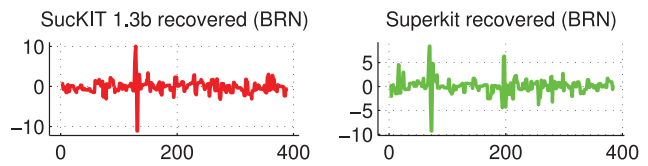

(b)
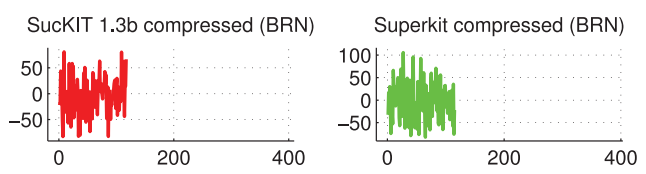

SucKIT 1.3b recovered (BRN)
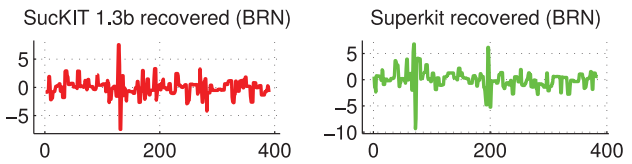

(c)
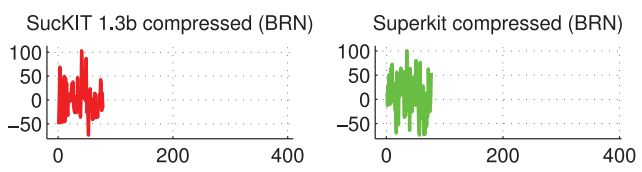

SucKIT $1.3 \mathrm{~b}$ recovered (BRN)
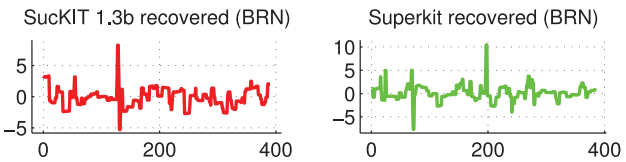

(d)
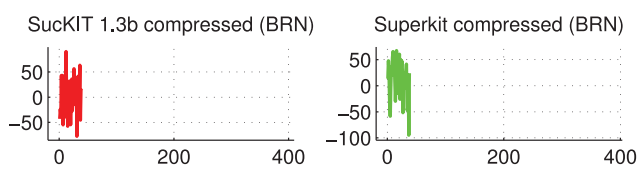

SucKIT 1.3b recovered (BRN)
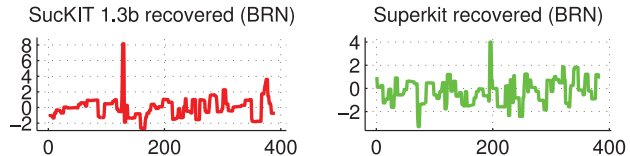
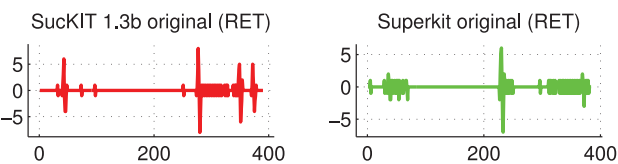

SucKIT 1.3b compressed (RET)

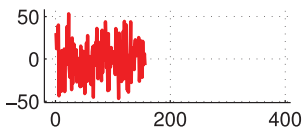

SucKIT 1.3b recovered (RET)

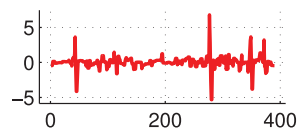

)
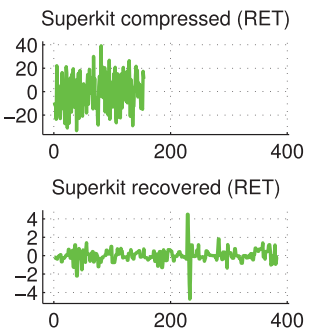

SucKIT 1.3b compressed (RET)

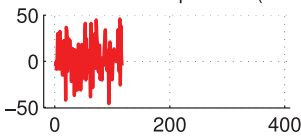

Superkit compressed (RET)

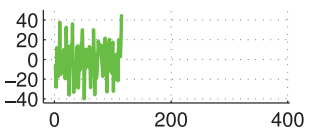

SucKIT 1.3b recovered (RET)
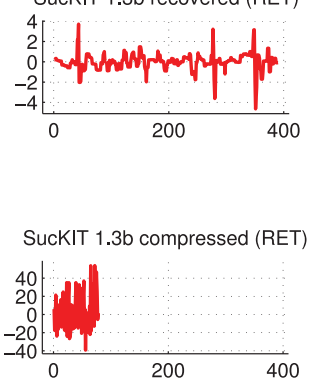

SucKIT 1.3b recovered (RET)
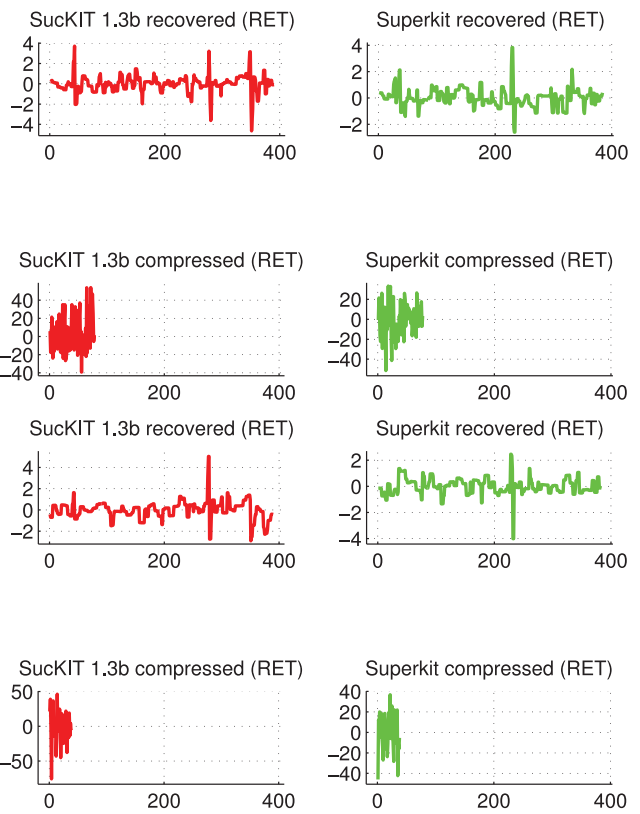

SucKIT 1.3b recovered (RET)
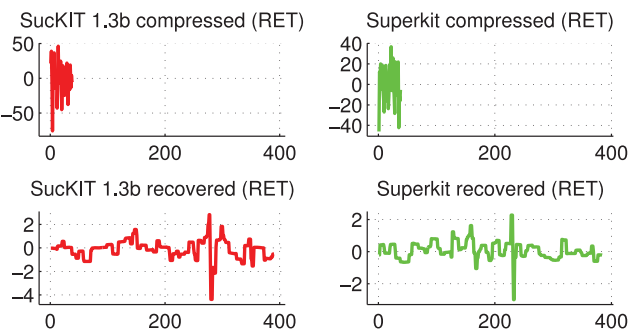

(e)

Fig. 9. The original BRN and RET profiles of SucKIT 1.3b and Superkit (a). The compressed and recovered profiles of the rootkits with the compression ratio of $0.4(\mathrm{~b}), 0.3(\mathrm{c}), 0.2(\mathrm{~d})$, and $0.1(\mathrm{e})$. 
Table IV. The Pearson's Correlation Coefficients Between the Recovered Profiles of Tested Rootkits and the Original Profiles of Reference Rootkits in the Database. Two Profiles with a Correlation Coefficient Greater than 0.70 Are Considered a Match. The Rootkits Cannot Be Identified from Their Recovered Profiles when the Compression Ratio Is Lower Than 0.2

\begin{tabular}{|c|c|c|c|c|c|c|c|c|c|}
\hline \multirow{2}{*}{$\begin{array}{c}\text { Rootkit under } \\
\text { test }\end{array}$} & \multicolumn{8}{|c|}{ Malware reference } & \multirow{2}{*}{$\begin{array}{c}\text { Identified } \\
\text { as }\end{array}$} \\
\hline & SucKIT 1.3b & Sk2rc2 & Superkit & Adore 0.42 & Phalanx b6 & Sebek 3.2 & Adore-ng & KBeast & \\
\hline SucKIT 1.3b-04 & 0.95 & 0.05 & 0.08 & 0.04 & 0.08 & 0.08 & 0.07 & 0.08 & SucKIT 1.3b \\
\hline SucKIT 1.3b-03 & 0.81 & 0.04 & 0.07 & 0.04 & 0.05 & 0.05 & 0.03 & 0.05 & SucKIT 1.3b \\
\hline SucKIT 1.3b-02 & 0.76 & 0.08 & -0.03 & 0.01 & -0.02 & 0.03 & -0.02 & -0.02 & SucKIT $1.3 \mathrm{~b}$ \\
\hline SucKIT 1.3b-01 & 0.34 & 0.04 & 0.03 & 0.02 & 0.03 & -0.01 & 0.01 & 0.01 & unknown \\
\hline Sk2rc2-04 & 0.11 & 0.87 & 0.01 & -0.03 & 0.07 & 0.09 & 0.12 & 0.11 & $\overline{~ S k 2 r c 2 ~}$ \\
\hline Sk2rc2-03 & -0.01 & 0.76 & -0.04 & 0.02 & -0.03 & 0.05 & 0.09 & 0.05 & Sk2rc2 \\
\hline Sk2rc2-02 & 0.07 & 0.71 & 0.04 & 0.04 & -0.02 & 0.04 & 0.05 & 0.03 & Sk2rc2 \\
\hline Sk2rc2-01 & 0.04 & 0.15 & 0 & 0.01 & 0.04 & 0.01 & 0.01 & 0.01 & unknown \\
\hline Superkit-04 & - -0.02 & 0.08 & $\overline{0.91}$ & -0.05 & 0.10 & 0.09 & 0.22 & 0.10 & Superkit \\
\hline Superkit-03 & 0.05 & 0.08 & 0.87 & 0.03 & 0.09 & 0.05 & 0.15 & 0.05 & Superkit \\
\hline Superkit-02 & 0.02 & 0.01 & 0.74 & 0.04 & 0.05 & 0.03 & 0.03 & 0.02 & Superkit \\
\hline Superkit-01 & 0.02 & -0.01 & 0.45 & 0 & -0.01 & 0.01 & -0.01 & -0.03 & unknown \\
\hline Adore 0.42-04 & 0.03 & 0.01 & -0.18 & 0.89 & 0.05 & 0.12 & 0.13 & 0.11 & Adore 0.42 \\
\hline Adore $0.42-03$ & 0.07 & 0.01 & -0.19 & 0.83 & 0.04 & 0.09 & 0.09 & 0.08 & Adore 0.42 \\
\hline Adore $0.42-02$ & -0.02 & 0.01 & -0.12 & 0.73 & 0.02 & 0.05 & 0.05 & -0.01 & Adore 0.42 \\
\hline Adore $0.42-01$ & 0.05 & 0.02 & 0.04 & 0.28 & -0.01 & -0.01 & 0.02 & 0.02 & unknown \\
\hline Phalanx b6-04 & 0.10 & 0.03 & 0.08 & 0.21 & 0.93 & $\overline{0.13}$ & 0.09 & 0.11 & Phalanx b6 \\
\hline Phalanx b6-03 & 0.05 & 0.05 & 0.05 & 0.12 & 0.88 & 0.09 & 0.05 & 0.08 & Phalanx b6 \\
\hline Phalanx b6-02 & 0.09 & -0.04 & -0.04 & 0.05 & 0.85 & 0.01 & 0.03 & 0.05 & Phalanx b6 \\
\hline Phalanx b6-01 & -0.04 & 0.01 & 0.03 & -0.01 & 0.62 & -0.02 & 0.01 & 0.02 & unknown \\
\hline Sebek 3.2-04 & -0.01 & 0.21 & 0.05 & 0.11 & 0.05 & 0.88 & 0.08 & 0.09 & Sebek 3.2 \\
\hline Sebek 3.2-03 & 0.08 & 0.11 & 0.04 & 0.05 & 0.03 & 0.83 & 0.06 & 0.05 & Sebek 3.2 \\
\hline Sebek 3.2-02 & 0.05 & 0.01 & -0.01 & 0.02 & 0.01 & 0.75 & 0.02 & 0.02 & Sebek 3.2 \\
\hline Sebek 3.2-01 & -0.03 & -0.03 & 0.02 & -0.01 & -0.02 & 0.55 & -0.01 & -0.01 & unknown \\
\hline Adore-ng-04 & 0.09 & 0.05 & 0.11 & 0.09 & 0.06 & 0.05 & 0.91 & 0.05 & Adore-ng \\
\hline Adore-ng-03 & 0.09 & 0.08 & 0.09 & 0.05 & 0.04 & -0.02 & 0.85 & 0.02 & Adore-ng \\
\hline Adore-ng-02 & -0.03 & 0.08 & 0.02 & 0.01 & 0.03 & -0.03 & 0.81 & 0.01 & Adore-ng \\
\hline Adore-ng-01 & -0.06 & 0.02 & -0.04 & -0.02 & 0.01 & 0.01 & 0.46 & 0.01 & unknown \\
\hline "KBeast-04 & 0.11 & 0.10 & 0.08 & 0.05 & 0.06 & 0.06 & 0.08 & 0.90 & KBeast \\
\hline KBeast-03 & 0.09 & 0.09 & 0.05 & 0.03 & 0.04 & 0.03 & -0.05 & 0.84 & KBeast \\
\hline KBeast-02 & -0.08 & 0.02 & 0.03 & 0.02 & -0.02 & 0.01 & 0.04 & 0.77 & KBeast \\
\hline KBeast-01 & 0.06 & -0.01 & 0.04 & 0.01 & -0.01 & -0.01 & -0.01 & 0.42 & unknown \\
\hline
\end{tabular}

correlation coefficient between a tested rootkit and its corresponding reference is above the threshold, meaning a successful identification. While with a compression ratio less than 0.2 , all the tested rootkits are considered as unknown malware. The identification fails because the accuracy of the recovery is too low.

\subsection{Performance Analysis}

In the following experiment, we perform the performance analysis in a monitored system for the proposed technique. We first evaluate the computational latency for compressing the original recorded HPC data with Compressive Sensing, which has to be done locally. This includes generating the sensing matrix and compressing the recorded data with the generated matrix. The evaluation is performed on the same platform described in Section 5.2.

In Compressive Sensing, the compression is performed by simply multiplying the sampled data by a sensing matrix, and therefore the compressing latency is relatively small. The execution times for compressing data in different sizes and with different compression ratios are shown in Figure 10(a). The result indicates that the execution 


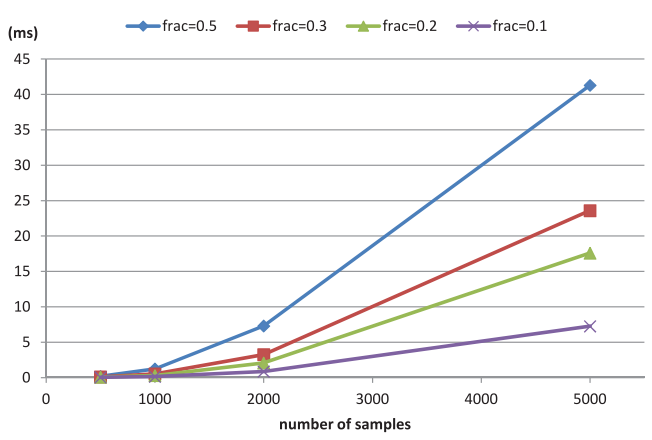

(a)

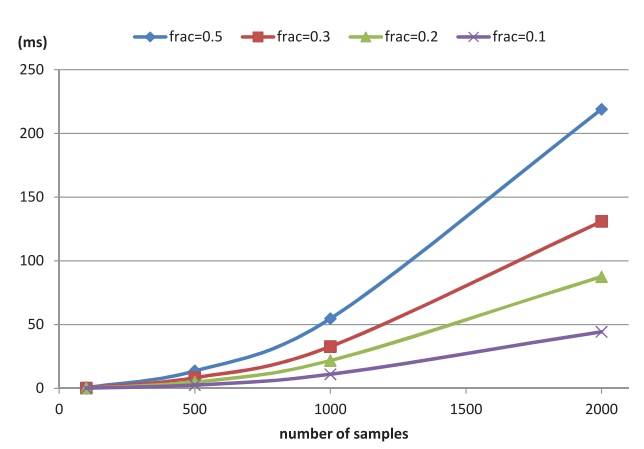

(b)

Fig. 10. (a) Computational latency for compressing sampled data with Compressive Sensing. (b) Computational latency for generating a sensing matrix. The execution times for both processes increase exponentially with the size of data to compress and increase linearly with the compression ratio.

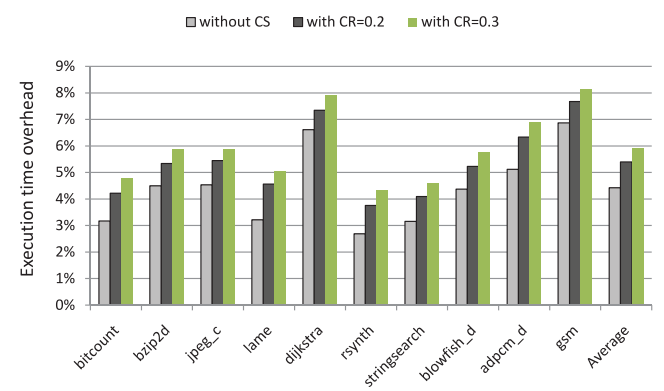

(a)

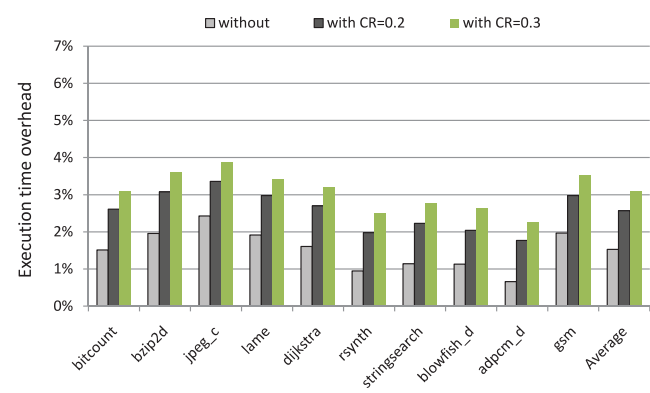

(b)

Fig. 11. Execution time overhead with and without Compressive Sensing when a periodical sampling is applied with an interval of 100,000 (a) and 300,000 (b) retired instructions. All the numbers are the average of 20 runs.

time increases exponentially with the size of data to compress and increases linearly with the compression ratio. For example, for a required compression ratio of 0.3 , compressing 500, 1000, and 2000 samples takes $0.113 \mathrm{~ms}, 0.539 \mathrm{~ms}$, and $3.26 \mathrm{~ms}$, respectively.

The computational latency for generating the sensing matrix is presented in Figure 10(b). As for compressing, the latency for generating the sensing matrix also depends on the compression ratio and the size of the data to compress. As shown in the results, generating a sensing matrix with the compression ratio of 0.3 for compressing $500,1000,2000$ samples costs $8.33 \mathrm{~ms}, 32.8 \mathrm{~ms}$, and $131 \mathrm{~ms}$, respectively. This latency is much larger than the latency for compression. However, the sensing matrix is only generated one time after the number of samples in each check and the compression ratio are determined. For the following checks with the same sample size and compression ratio, the generated sensing matrix can be simply reused. So it has very little impact on the runtime latency in a monitored system.

In Figure 11, we compare the execution time for the 10 benchmark applications when a periodical sampling is applied with a interval of 100,000 and 300,000 retired instructions. We also measure the additional overhead due to Compressive Sensing with compression ratio of 0.3 and 0.2 . When a sampling interval of 100,000 retired instructions is applied, the average execution time overhead is $4.42 \%$ without Compressive Sensing and is $5.40 \%$ and $5.92 \%$ with the compression ratio of 0.3 and 0.2 ; 
when the sampling interval increases to 300,000 retired instructions, the average execution time overhead is $1.53 \%$ without Compressive Sensing and is $2.57 \%$ and $3.09 \%$ with the compression ratio of 0.3 and 0.2 , respectively.

The operation in the remote sever contains two main steps: recovering the compressed data to an estimated full profile and performing the malware detection and identification based on the recovered full profile. We use a platform with a $2.53-\mathrm{GHz}$ Intel Core 2 Duo P8700 CPU to run the remote server operations. The HPC profile recovery step takes $112 \mathrm{~ms}, 265 \mathrm{~ms}$, and $786 \mathrm{~ms}$ with the original data size of 500 , 1000 , and 2000 samples, respectively. For the malware detection and identification, we run the One-Class Support Vector Machine (OC-SVM) classifier to determine if a runtime collected sample is fitted into the pregenerated "golden" model. The execution time for the runtime classification is about $50 \mathrm{~ms}$. In a real case, the remote server is usually configured with more computational resources than the platform we use in the evaluation. It can be expected that the latency could be significantly reduced.

\section{RELATED WORK}

HPC-based integrity checking and malware detection. The scheme proposed in Malone et al. [2011] uses HPCs for integrity checking of programs. It targets malicious modifications to the user space programs. CFIMon [Xia et al. 2012] leverages the branch trace store mechanism in performance counters for the purpose of detecting attacks to control-flow, including classic code-injection attacks and emerging code-reuse attacks. Tang et al. [2014] uses unsupervised machine learning on microarchitectural features to detect malware. It shows that the execution of a program under common attacks can be characterized with the stream of hardware event measurements which are easily accessible from the HPC. These techniques assume the OS kernel is trusted. Demme et al. [2013] leverages HPCs to collect fine-grained hardware event-based traces of Android malware and Linux kernel rootkits on ARM and Intel platforms. It then applies machine learning classification algorithms to detect variants of known malware. A hardware support is also proposed to prevent subversion of the protection scheme. NumChecker [Wang and Karri 2016] is a virtualization-based framework to detect and identify control-flow modifying kernel rootkits in a guest Virtual Machine (VM). It detects malicious modifications to a system call in the guest VM by monitoring the occurrences of hardware events during the system call's execution. HPCs are leveraged to automatically count the events. Ozsoy et al. [2015] proposes an always-on hardware malware detection engine integrated at the commit stage of a conventional processor. The detection engine collects subsemantic features, including the occurrences of lowlevel hardware events for online malware detection. ConFirm [Wang et al. 2015] is a low-cost technique that detects malicious modifications to the firmware of embedded control systems by monitoring the hardware events using HPCs.

Compressive Sensing in Cyber Security. Applying Compressive Sensing technology to cyber security is a new research topic and has not been studied much. To the best of our knowledge, DISTROY [Gwon et al. 2011] is the only effort that has been made so far. It leverages Compressive Sensing to reduce the I/O requirement in detecting small power leakage due to Trojans embedded in ICs. With reduced I/O, the approach still allows off-chip Trojan detectors to recover the most significant indicators of Trojan-induced power variations. DISTROY enables simple encoding and accurate reconstruction of the most significant power consumption anomalies among the test vectors applied. It can detect the presence of power leakage resulting from the background power consumption of on-chip Trojans even when the drawn power is extremely small. 


\section{CONCLUSION AND FUTURE WORK}

In this article we present a new HPC-based malware detection and identification framework. To reduce the overhead in the monitored systems with limited storage and computing resources, instead of being analyzed locally, the sampled HPC data is sent to a remote analyzer with much more computing capability for malware detection and identification. To minimize the I/O bandwidth required for transmission, the fine-grained HPC profiles are compressed into much smaller vectors with the technique called Compressive Sensing before being sent out through the network. The compressed version of the sampled data is then accurately recovered to an estimated full profile by the remote analyzer for further malware detection and identification.

Our future research will focus on determining optimal recovery algorithms which have better balance between speed and performance. Also, a more effective and comprehensive classification algorithm for robust profile matching should be explored. Moreover, using Compressive Sensing for reducing the data deluge will be evaluated on HPC-based detections on other malware besides kernel rootkits.

\section{REFERENCES}

Martín Abadi, Mihai Budiu, Ulfar Erlingsson, and Jay Ligatti. 2005. Control-flow integrity. In Proceedings of the 12th ACM Conference on Computer and Communications Security. 340-353.

Shawkat Ali and Kate A. Smith. 2006. On learning algorithm selection for classification. Appl. Soft Comput. 6 (2006), 119-138.

T. Asha, U. M. Shravanthi, N. Nagashree, and M. Monika. 2013. Building machine learning algorithms on Hadoop for bigdata. Int. J. Eng. Technol. 3, 2 (2013), 143-147.

Thomas Ball and James R. Larus. 2000. Using paths to measure, explain, and enhance program behavior. IEEE Trans. Comput. 33, 7 (2000), 57-65.

Richard G. Baraniuk. 2007. Compressive sensing. Lect. Not. IEEE Signal Process. Mag. 24, 4 (2007), $118-121$.

Emmanuel J. Candès. 2008. The restricted isometry property and its implications for compressed sensing. Compt. Rend. Math. 346, 9 (2008), 589-592.

cBench. 2010. Collective Benchmark (cBench). Retrieved from http://ctuning.org/wiki/index.php/CTools: CBench.

John Demme, Matthew Maycock, Jared Schmitz, Adrian Tang, Adam Waksman, Simha Sethumadhavan, and Salvatore Stolfo. 2013. On the feasibility of online malware detection with performance counters. In Proceedings of the 40th Annual International Symposium on Computer Architecture. 559-570.

Jiaqing Du, Nipun Sehrawat, and Willy Zwaenepoel. 2011. Performance profiling of virtual machines. ACM SIGPLAN Not. 46, 7 (2011), 3-14.

EEI-AEIC-UTC. 2011. Smart meters and smart meter systems: A metering industry perspective. Retrieved from http://www.eei.org/issuesandpolicy/grid-enhancements/Documents/smartmeters.pdf.

Yonina C. Eldar and Gitta Kutyniok. 2012. Compressed Sensing: Theory and Applications. Cambridge University Press, Cambridge.

A. Murat Fiskiran and Ruby B. Lee. 2004. Runtime execution monitoring (REM) to detect and prevent malicious code execution. In Proceedings of the 22nd International Conference on Computer Design. $452-457$.

Irina F. Gorodnitsky and Bhaskar D. Rao. 1997. Sparse signal reconstruction from limited data using FOCUSS: A re-weighted minimum norm algorithm. IEEE Trans. Signal Process. 45, 3 (1997), 600616.

Youngjune L. Gwon, H. T. Kung, and Dario Vlah. 2011. DISTROY: Detecting integrated circuit Trojans with compressive measurements. In Proceedings of 6th USENIX Workshop on Hot Topics in Security.

Intel Inc. 2010. Intel VTune Performance Analyzer. Retrieved from http://software.intel.con/en-us/ intel-vtune.

Tapas Kanungo, David M. Mount, Nathan S. Netanyahu, Christine D. Piatko, Ruth Silverman, and Angela Y. Wu. 2002. An efficient k-means clustering algorithm: Analysis and implementation. IEEE Trans. Pattern Anal. Machine Intell. 24, 7 (2002), 881-892.

John Levon and Philippe Elie. 2010. Oprofile: A system profiler for Linux. Retrieved from http://oprofile. sourceforge.net. 
Chengbo Li, Wotao Yin, and Yin Zhang. 2009. TVAL3: TV Minimization by Augmented Lagrangian and Alternating Direction Algorithm. Retrieved from http://www.caam.rice.edu/ optimization/L1/TVAL3/.

David Lie, Chandramohan Thekkath, Mark Mitchell, Patrick Lincoln, Dan Boneh, John Mitchell, and Mark Horowitz. 2000. Architecturual support for copy and tamper resistant software. In Proceedings of 9th International Conference on Architectural Support for Programming Languages and Operating Systems. 168-177.

Hsuan-Tien Lin. 2008. Introduction to Adaptive Boosting. Retrieved from http://www.csie.ntu.edu. tw/ htlin/course/ml08fall/doc/adaboost.pdf.

Linux. 2010. Performance Counters for Linux. Retrieved from http://lwn.net/Articles/310176.

K. Berker Loğoğlu and Tuğrul K. Ateş. 2010. Speeding-up Pearson correlation coefficient calculation on graphical processing units. In Proceedings of 18th IEEE Signal Processing and Communications Applications Conference. 840-843.

Corey Malone, Mohamed Zahran, and Ramesh Karri. 2011. Are hardware performance counters a cost effective way for integrity checking of programs? In Proceedings of the 6th ACM Workshop on Scalable Trusted Computing. 71-76.

Meltem Ozsoy, Caleb Donovick, Iakov Gorelik, Nael Abu-Ghazaleh, and Dmitry Ponomarev. 2015. Malwareaware processors: A framework for efficient online malware detection. In Proceedings of the 21st International Symposium on High Performance Computer Architecture. 651-661.

Philip Schniter, Lee C. Potter, and Justin Ziniel. 2008. Fast Bayesian matching pursuit. In Proceedings of Information Theory and Applications Workshop. 326-333.

Timothy Sherwood, Erez Perelman, Greg Hamerly, Suleyman Sair, and Brad Calder. 2003. Discovering and exploiting program phases. IEEE Micro 23, 6 (2003), 84-93.

Stress. 2010. Stress project. Retrieved from http://people.seas.harvard.edu/ apw/stress/.

G. Edward Suh, Charles W. O'Donnell, Ishan Sachdev, and Srinivas Devadas. 2005. Design and implementation of the AEGIS single-chip secure processor using physical random functions. In Proceedings of 32nd Annual International Symposium on Computer Architecture. 25-36.

M. G. Matt Syal and Kweku Ofei-Amoh. 2013. Smart-grid technologies in housing. Cityscape 15, 2 (2013).

Adrian Tang, Simha Sethumadhavan, and Salvatore Stolfo. 2014. Unsupervised anomaly-based malware detection using hardware features. In Proceedings of 17th International Symposium on Research in Attacks, Intrusions and Defenses. 109-129.

Joel Tropp and Anna Gilbert. 2007. Signal recovery from random measurements via orthogonal matching pursuit. IEEE Trans. Inform. Theor. 53, 12 (2007), 4655-4666.

Xueyang Wang and Ramesh Karri. 2013. Numchecker: Detecting kernel control-flow modifying rootkits by using hardware performance counters. In Proceedings of the 50th Design Automation Conference. 1-7.

Xueyang Wang and Ramesh Karri. 2016. Re-using hardware performance counters to detect and identify kernel control-flow modifying rootkits. IEEE Transactions on Computer-Aided Design of Integrated Circuits and Systems 35, 3 (2016), 485-498.

Xueyang Wang, Charalambos Konstantinou, Michail Maniatakos, and Ramesh Karri. 2015. ConFirm: Detecting firmware modifications in embedded systems using hardware performance counters. In Proceedings of the 34th International Conference on Computer-Aided Design. 544-551.

Rebecca M. Willett, Zachary T. Harmany, and Roummel F. Marcia. 2010. Poisson image reconstruction with total variation regularization. In Proceedings of the 17th IEEE International Conference on Image Processing. 4177-4180.

Yubin Xia, Yutao Liu, Haibo Chen, and Binyu Zang. 2012. CFIMon: Detecting violation of control flow integrity using performance counters. In Proceedings of the 42nd Annual International Conference on Dependable Systems and Networks. 1-12.

Feng Xue. 2008. Attacking the Antivirus. Retrieved from www.blackhat.com/presentations/bh-europe.../ bh-eu-08-xue-WP.pdf.

Received February 2015; revised November 2015; accepted December 2015 